\title{
Chiral symmetry on a lattice with hopping interactions
}

\author{
Takanori Sugihara ${ }^{\mathrm{a} *}$

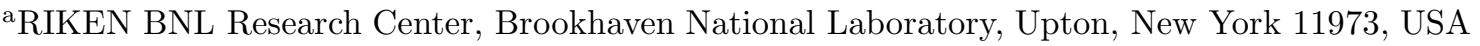

The species doubling problem of the lattice fermion is resolved by introducing hopping interactions that mix left- and right-handed fermions around the momentum boundary. Approximate chiral symmetry is realized on the lattice. The deviation of the fermion propagator from the continuum one is small.

\section{INTRODUCTION}

In contrast with the great success of lattice gauge theory, lattice fermions remain a longstanding problem. Naive discretization causes the species doubling problem 1 . The situation does not change regardless of how the lattice spacing is reduced as long as the space-time derivative is modeled as a naive difference. Many attempts have been made to fix the doubling problem 1234567 . Wilson removed doublers at low energy by introducing an interaction that mixes left- and right-handed fermions 1 . However, unwanted degeneracy persist at high energy and chiral symmetry is explicitly broken. To fix these problems, Kaplan modified Wilson's fermion with an extra dimension 4 and succeeded in realizing approximate chiral symmetry. However, the cost of numerical calculations based on it is not cheap. If we find a method to perform such calculations without the extra dimension, calculation time decreases largely and a deeper understanding of quantum field theory becomes possible.

The lattice fermion has another serious problem. The fermion propagator defined on a lattice deviates from the continuum one even if the doublers are removed with the existing techniques such as Kaplan's fermion [5]. We need to modify the discretized propagator somehow so that it is close to the continuum one as far as possible.

In general, the extra dimension can be expressed as hopping interactions in a lowerdimensional system. Kaplan's fermion is a formulation with an extra dimension, so there must be a

*sugihara@bnl.gov corresponding Hamiltonian with no extra dimension. Also, the shape of the fermion propagator can be improved with hopping interactions.

In this talk, based on a Hamiltonian formalism, we introduce ultralocal hopping interactions to remove doublers and improve momentum dependence of fermion energy. (The word "ultralocal" means that fermion hopping is restricted to a finite range on a real-space lattice 8].) From knowledge of the continuum theory, we know the correct momentum dependence of the energy. We start from momentum space and go back to real space by way of discrete Fourier transform. See Ref. 9 for the details of this work.

\section{FORMULATION}

Let us consider a free Dirac fermion on a $(1+1)$ dimensional Hamiltonian lattice.

$H=\frac{1}{a} \sum_{l=-N / 2+1}^{N / 2} p_{l} \bar{\zeta}_{l} \gamma^{1} \zeta_{l}$,

where $l$ is an index for momentum and $N$ is the number of sites. $\zeta_{l}$ and $\bar{\zeta}_{l}$ are discrete Fourier transform of real-space two-component fermion operators $\psi_{n}$ and $\bar{\psi}_{n} \equiv \psi_{n}^{\dagger} \gamma^{0}$ for $n=1,2, \ldots, N$, respectively. The system is quantized with anticommutators in the usual way. Periodic boundary conditions are assumed in real space, $p_{l} \equiv$ $2 \pi l / N$. We try to create a real-space Hamiltonian with no doubler that reproduces Eq. (1). To find real-space representation of $p_{l}$, let us consider the 
following function

$$
\sum_{\alpha=1}^{M} \frac{2(-1)^{\alpha-1}}{\alpha} \sin \alpha p .
$$

In the limit $M \rightarrow \infty$, the function (2) goes to $p$. The function (2) necessarily has a node at the boundary $p= \pm \pi$ because it has a periodicity of $2 \pi$. The node is the cause of the doubling problem. The doubler remains as a singularity at the boundary even if the limit $M \rightarrow \infty$ is taken (by "doubler" we mean unwanted energy degeneracy that is not contained in the continuum theory). Anyway, the parameter $M$ needs to be small for practical formulation because $M$ corresponds to the maximum distance of fermion hopping.

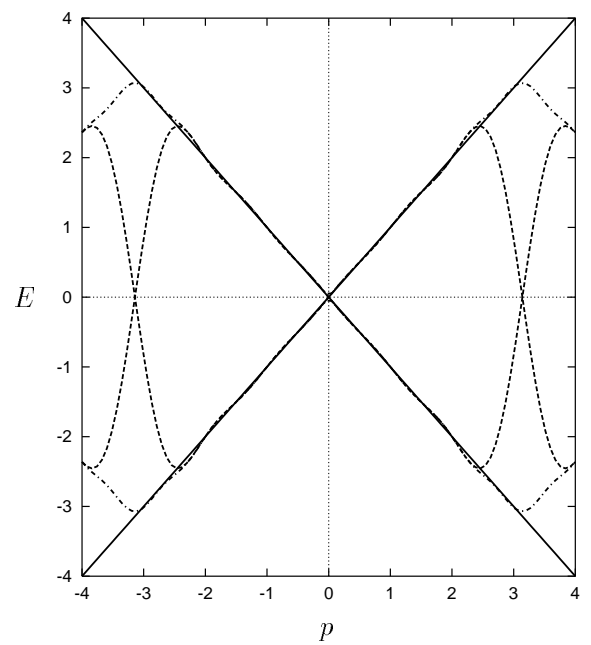

Figure 1. The solid line plots the correct energy $\pm p$ from the continuum theory. The dashed line plots the function $\pm s(p)$ for $M=5$ with the Lanczos factor. The dot-dashed line plots the function $\pm k(p)$ for $M=5, u=130$, and $v=8.4$ with the Lanczos factor. The function $\pm k(p)$ almost agrees with $\pm p$ in the fundamental region $|p| \leq \pi$ except for a small deviation around momentum $|p| \sim 2.3$.
In addition to the doubler around the momentum boundary, the function (2) has another degeneracy. It oscillates around $p$ and has local minima if the summation is truncated with a small $M$. In Fourier analysis, it is called the Gibbs phenomenon, which occurs if a function to be expanded has a singularity [10]. The oscillation can be removed by replacing Eq. (2) with

$s(p)=\sum_{\alpha=1}^{M} S_{\alpha} \sin \alpha p$,

where

$S_{\alpha} \equiv F_{\alpha} \frac{2(-1)^{\alpha-1}}{\alpha}, \quad F_{\alpha} \equiv \frac{M+1}{\pi \alpha} \sin \left(\frac{\pi \alpha}{M+1}\right)$.

$F_{\alpha}$ is called the Lanczos factor [10. The factor almost removes the oscillation of Eq. (2). However, the doubler modes around the boundary still remain. We are going to remove them by a trick with hopping interactions. Let us consider the following momentum-space Hamiltonian:

$H=\sum_{l=-N / 2+1}^{N / 2}\left(s_{l} \bar{\zeta}_{l} \gamma^{1} \zeta_{l}+m \bar{\zeta}_{l} \zeta_{l}\right)$,

where $s_{l} \equiv s\left(p_{l}\right) / a$ and $m$ is fermion mass. We introduce interactions $c_{l}$ that mix left- and righthanded fermions

$H=\sum_{l=-N / 2+1}^{N / 2} \zeta_{l}^{\dagger}\left(\begin{array}{cc}s_{l} & m+c_{l} \\ m+c_{l} & -s_{l}\end{array}\right) \zeta_{l}$,

where $c_{l}$ are assumed to be nonzero only for $|l| \sim$ $N / 2$. The Hamiltonian (5) can be diagonalized.

$H=\sum_{l=-N / 2+1}^{N / 2} \zeta_{l}^{\prime \dagger}\left(\begin{array}{cc}k_{l} & 0 \\ 0 & -k_{l}\end{array}\right) \zeta_{l}^{\prime}$,

where $k_{l} \equiv \sqrt{s_{l}^{2}+\left(m+c_{l}\right)^{2}}$ are energies of one particle states and $\zeta_{l}^{\prime}$ are transformed variables. The coefficients $c_{l}$ are determined so that energy of one-particle states becomes close to the continuum one as far as possible.

In Fig. 10 the functions $\pm p, \pm s(p)$, and $\pm k(p)$ are compared, where $k(p) \equiv \sqrt{s^{2}(p)+c^{2}(p)}$. The function $\pm k(p)$ corresponds to energy of oneparticle states given by the Hamiltonian (5i) with 
$m=0$. The function $\pm k(p)$ agrees well with the correct energy $\pm p$ from the continuum theory in the fundamental region $|p| \leq \pi$. The Hamiltonian (5) approximately reproduces the continuum theory without doubler if we identify $c_{l}=c\left(p_{l}\right) / a$ and $k_{l}=k\left(p_{l}\right) / a$.

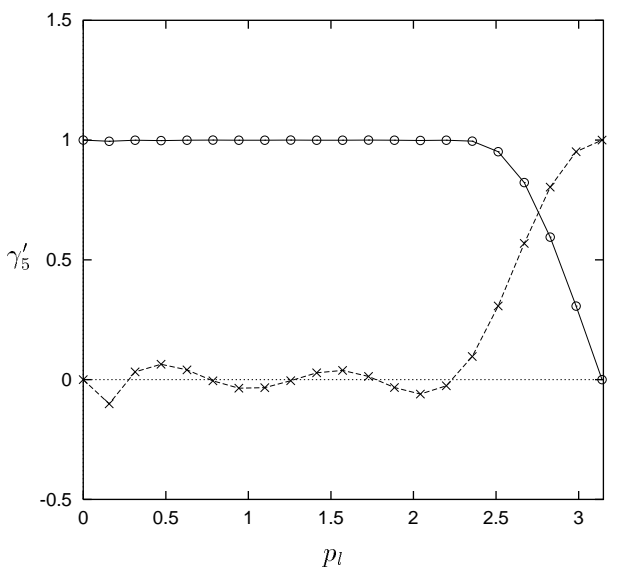

Figure 2. The matrix elements of $\gamma_{5}^{\prime}$ are plotted as functions of the momentum $p_{l}$ for $a=1$, $M=5, u=130$, and $v=8.4$ with the Lanczos factor. For a lattice size $N=40$, the functions are plotted in the right-half plane of momentum space $p_{l} \geq 0$. The circles are plots of the diagonal $(1,1)$ element of $\gamma_{5}^{\prime}$. The crosses are plots of minus of the off-diagonal $(1,2)$ element of $\gamma_{5}^{\prime}$. The solid and dashed lines are plotted to guide the eyes.

Figure 2 shows the diagonal and off-diagonal matrix elements of the transformed $\gamma_{5}$ in the new basis that diagonalizes the Hamiltonian (5) with $m=0$. The diagonal $(1,1)$ element of $\gamma_{5}^{\prime}$ is almost unity at low and intermediate energy $p_{l}<2.3$ and deviates from unity at $p_{l}>2.3$. The offdiagonal $(1,2)$ element of $\gamma_{5}^{\prime}$ oscillates around zero at $p_{l}<2.3$ and becomes unity at $p_{l}=\pi$. At low energy, the deviation of the off-diagonal elements from zero is not large and becomes smaller as the parameter $M$ increases. At $p_{l}<2.3$, the transformed left- and right-handed fermions have approximately the correct chiral charges 1 and -1 , respectively. The low-energy Hamiltonian (5) has approximate chiral symmetry because the commutation relation between the Hamiltonian (5) and chiral charge defined with $\gamma_{5}^{\prime}$ is almost zero for small $l$. The errors associated with chiral symmetry can be improved in a systematic way by increasing $M$. The value used here for the parameter $M$ is sufficiently small and does not deny application of the model to actual numerical analysis with a computer.

The real-space Hamiltonian and the Euclidean action for the Dirac spinor is obtained by substituting the discrete Fourier transform into Eq. (15). As usual, gauge symmetry can be implemented by inserting exponentiated gauge fields between the hopping fermions. The continuum limit $a \rightarrow 0$ is taken with $M$ fixed. The method can be extended to higher-dimensional cases by taking care of the doubler of each direction in the same way.

In this talk, I have shown how to realize approximate chiral symmetry at low energy. Explicit breaking of chiral symmetry has been compressed to high energy. In future works, it should be precisely checked if insertion of gauge interactions affects chiral properties.

\section{REFERENCES}

1. K. G. Wilson, in New Phenomena in Subnuclear Physics, Erice, 1975 edited by A. Zichichi (Plenum, New York, 1977).

2. J. Kogut and L. Susskind, Phys. Rev. D 11, 395 (1975).

3. S. D. Drell, M. Weinstein, and S. Yankielowicz, Phys. Rev. D 14, 487 (1976).

4. D. B. Kaplan, Phys. Lett. B 288, 342 (1992); M. F. Golterman, K. Jansen and D. B. Kaplan, ibid. 301, 219 (1993)

5. M. Creutz and I. Horvath, Phys. Rev. D 50, 2297 (1994).

6. H. Neuberger, Phys. Lett. B 417, 141 (1998); 427, 353 (1998).

7. M. Luscher, Phys. Lett. B 428, 342 (1998).

8. W. Bietenholz, Eur. Phys. J. C 6, 537 (1999).

9. T. Sugihara, Phys. Rev. D 68, 034502 (2003). 
10. G. B. Arfken and H. J. Weber, Mathematical Methods for Physicists, (Academic Press, New York, 1995). 